\title{
Residence Time Distribution and Hold-up in a Cocurrent Upflow Packed Bed Reactor at Elevated Pressure
}

\author{
Klaas B. van Gelder and K. Roel Westerterp* \\ Dedicated to Professor Ewald Wicke on the occasion of his 75th birthday
}

\begin{abstract}
The residence time distribution in liquid phase was measured in a cocurrent upflow packed bed reactor for the system methanol-hydrogen at low Reynolds numbers and at elevated pressure. The plug flow with axial dispersion model was used to describe mixing in the system. The imperfect pulse method was used to measure the system response to a tracer pulse input. The parameters were calculated using the weighted moments method. The influence of the weighting factor was investigated. The experimental and theoretical outputs, as calculated by convolution, agreed very well. Different types of correlations were used for the Bodenstein number and liquid hold-up. From these correlations, the optimal one was selected for each parameter. A comparison was made between the ordinary moments and the weighted moments methods which led to the conclusion that the latter method is superior with respect to the accuracy of the estimated parameters and therefore strongly recommended.
\end{abstract}

\section{Introduction}

In our laboratory, we investigate hydrogenation reactions of chemicals dissolved in methanol in a cocurrent upflow packed bed reactor at elevated pressure. It is the purpose of this paper to present data and correlations for the hold-up and mixing in such a reactor. In view of the required long residence times for the liquid phase, gas and liquid loads are relatively low. Several correlations have been presented in literature for the prediction of hold-up and mixing in cocurrent upflow packed bed reactors as functions of flow conditions. Excellent reviews were given by Shah [1] and Hoffmann [2]. A summary of correlations presented for the hold-up is given in Table 1 and for the Bodenstein number in Table 2.

Most of these correlations were derived at atmospheric pressure, for the system air/water and at moderate to large liquid- and gas-phase Reynolds numbers. The correlations are based on superficial velocities, Reynolds numbers or mass fluxes and differ distinctly in the way they correlate the hold-up or mixing as functions of flow conditions.

In the correlations for $\varepsilon_{\mathrm{G}}$, the exponent on $U_{\mathrm{L}}$ varies from 0 to 0.2 and that on $U_{\mathrm{G}}$ from -0.2 to +0.3 . For the Bodenstein number, we find that the exponent on $U_{\mathrm{L}}$ ranges between +0.25 and +0.96 and that on $U_{\mathrm{G}}$ between -0.48 and -0.16 . In our opinion, such a large variation of the exponents indicates that the studied phenomena cannot be adequately correlated simply by a product of certain powers of dimensionless groups.

\section{The Plug Flow with Axial Dispersion Model}

The plug flow with axial dispersion model (PD-Model) was used to describe mixing in our system. This model essentially

\footnotetext{
* Dr. Ir. K.B. van Gelder and Prof. Dr. Ir. K.R. Westerterp, University of Twente, Department of Chemical Engineering, P.O. Box 217, NL-7500 AE Enschede.
}

assumes plug flow in the liquid-phase. Mixing is characterized by a simple one-dimensional axial dispersion coefficient $D_{\mathrm{ax}}$ of Fick's law type. The differential equation describing mixing in dimensionless form is ${ }^{1)}$

$\frac{\partial C}{\partial \theta}=\frac{1}{\operatorname{Pe}} \frac{\partial^{2} C}{\partial Z^{2}}-\frac{\partial C}{\partial Z}$,

where $\theta$ is the dimensionless time $t / \tau, Z$ dimensionless reactor length $z / L, L$ length of the reactor, Pe dimensionless parameter governing the degree of mixing $\mathrm{Pe}=U_{\mathrm{L}} L / D_{\mathrm{ax}}, U_{\mathrm{L}}$ actual liquid velocity and $C$ the dimensionless concentration $c / c_{0}$.

The PD-Model characterizes mixing by only one parameter and is therefore the simplest differential model, which accounts for its wide use. Depending on the boundary conditions (open or closed system boundaries), different solutions are obtained for Eq. (1).

\section{The Boundary Problem}

The solution of Eq. (1) depends on the applied boundary conditions. The correct form of the boundary conditions is governed by the conditions in the inlet and outlet of the system under investigation. Two types of boundaries can be distinguished, i.e. open and closed. A closed boundary implies that a molecule can pass the boundary only in one direction. Thus, a tracer molecule can enter the system only through the entrance boundary and can leave the system only through the exit boundary. An open boundary, on the other hand, allows the molecules to pass the boundary several times in opposite directions, thus enabling them to spend some time outside the system. The measured residence time is then a function of the total time, i.e. spent both inside and outside the system. These excursions outside the system complicate the situation because a detection device can

1) List of symbols at the end of the paper. 
Table 1. Hold-up correlations from literature.

\begin{tabular}{llll}
\hline Reference & System & Packing & Proposed correlation \\
\hline Weber [23] & Air/Water & 5 mm spheres & $h_{0 \mathrm{G}}=0.079 U_{0 \mathrm{G}}^{0.3}$ \\
Weber [23] & Air $+2 \%$ EtOH/Water & $5 \mathrm{~mm}$ spheres & $h_{0 \mathrm{G}}=0.152 U_{0 \mathrm{G}}^{0.2}$ \\
Weber [23] & Air/Water & $2 \mathrm{~mm}$ spheres & $h_{0 \mathrm{G}}=0.078 U_{0 \mathrm{G}}^{\mathbf{0 . 2 4}}$ \\
Weber [23] & Air $+2 \%$ EtOH/Water & $2 \mathrm{~mm}$ spheres & $h_{0 \mathrm{G}}=0.113 U_{0 \mathrm{G}}^{\mathbf{0 . 3}}$ \\
Weber [23] & Air/Water & $4 \times 10 \mathrm{~mm}$ cylinders & $h_{0 \mathrm{G}}=0.079 U_{\mathrm{OG}}^{0.33}$ \\
Turpin, Huntington [22] & Air/Water & various tubular particles & $h_{\mathrm{L}}=-0.035+0.182\left(G_{\mathrm{L}} / G_{\mathrm{G}}\right)^{0.24}$ \\
Stiegel, Shah [21] & Air/Water & $2.8 \times 5.6$ and 3.1 $\times 3.1 \mathrm{~mm}$ & $h_{\mathrm{L}}=1.47 \operatorname{Re}_{\mathrm{G}}^{0.11} \operatorname{Re}_{\mathrm{L}}^{-0.19}\left(a_{\mathrm{s}} d_{\mathrm{s}}\right)^{-0.41}$ \\
& & cylinders & \\
Ford [24] & Air/Water & \pm 1 mm particles & $h_{\mathrm{G}}=0.212\left(\operatorname{Re}_{\mathrm{G}} / \operatorname{Re}_{\mathrm{L}}\right)^{0.2}\left(\mu_{\mathrm{L}} / \mu_{\mathrm{G}}\right)^{0.24}$ \\
Achwal, Stepanek [25] & Air/Water & 6mm cylinders & $1 / h_{\mathrm{G}}=1+4.33 U_{0 \mathrm{~L}}^{0.433}\left(U_{0 \mathrm{~L}} / U_{0 \mathrm{G}}\right)^{0.563}$ \\
Saada [26] & Air/Water & glass ballotini & $h_{\mathrm{L}}=0.48\left(\operatorname{Re}_{\mathrm{G}} / \operatorname{Re}_{\mathrm{L}}\right)^{0.25}$ \\
& & spheres of several diameters & \\
\hline
\end{tabular}

Table 2. Bodenstein number correlations from literature

\begin{tabular}{|c|c|c|c|}
\hline Reference & System & Packing & Proposed correlation \\
\hline Weber [23] & Air/Water & $\begin{array}{l}5 \mathrm{~mm} \text { spheres } \\
4 \times 10 \mathrm{~mm} \text { cylinders } \\
6.2 \mathrm{~mm} \text { Raschig rings }\end{array}$ & $\begin{array}{l}\mathrm{Bo}_{\mathrm{L}}=0.12 X_{0}^{0.48} \\
\mathrm{Bo}_{\mathrm{L}}=0.024 X_{0}^{0.46} \\
\mathrm{Bo}_{\mathrm{L}}=0.017 X_{0}^{0.43} \\
\text { where } X_{0}=\left(U_{\mathrm{L}} / U_{\mathrm{G}}\right) \operatorname{Re}_{\mathrm{G}} \mathrm{Sc}_{\mathrm{L}}\end{array}$ \\
\hline $\begin{array}{l}\text { Stiegel \& Shah } \\
\text { [21] }\end{array}$ & Air/Water & $\begin{array}{l}2.8 \times 5.6 \text { and } \\
3.1 \times 3.1 \mathrm{~mm} \text { cylinders }\end{array}$ & $\mathrm{Bo}_{\mathrm{L}}=0.128 \operatorname{Re}_{\mathrm{G}}^{0.245} \operatorname{Re}_{\mathrm{L}}^{-0.16}\left(a_{\mathrm{s}} d_{\mathrm{eq}}\right)^{0.53}$ \\
\hline
\end{tabular}

only register a passing molecule but cannot tell whether it is leaving or (re-)entering the system. A pulse injected at the entrance boundary for instance would travel partially outside the system before entering it through the entrance boundary. As a consequence, the pulse response function derived for a closed/closed system does not apply to an open/open system. For an open/open system, a transfer function, which transforms the concentration curve at the entrance to that at the exit, has to be used. The open/open system can then be treated similarly to a closed/closed system.

Gibilaro [3] and Nauman [4] examined the problems associated with open boundaries in detail. The conclusion which can be drawn from their papers is that an open/open system has the same RTD function as a closed/closed system if the time the tracer molecules spent outside the system boundaries is excluded. This means that, when using residence time distribution functions to calculate conversions in chemical reactors, the pulse response function derived for the closed/closed case should be used, because the time spent outside the reactor bed does not contribute to the conversion (at least in the case of heterogeneous catalytic reactions)! On the other hand, for the calculation of the parameters $\mathrm{Pe}$ and $\tau$, the solution for the open/open case should be used.

A summary of several solutions for combinations of the two types of boundaries, as found in literature, is given in Table 3 .
No explicit time domain solution was found for an open/closed system, so that we had to derive it ourselves. This could be readily achieved on the basis of the work of Wen and Fan [5] and of Villermaux and van Swaaij [6]. Wen and Fan give the transfer function in the Laplace domain for all four possible combinations of entrance and exit boundaries. Villermaux and van Swaaij give the transfer function in the time domain for their model of plug flow with axial dispersion and mass transfer between flowing and stagnant regions. In the case of no mass transfer, their model reduces to the PD-Model with closed/open system boundaries. In this case, the transfer function in the Laplace domain is the same as that given by Wen and Fan for the open/closed system. Therefore, the solution for the closed/open system of Villermaux and van Swaaij also applies to the open/closed system. Apparently, it is immaterial which of the two boundaries is open and which is closed.

The authors of the second solution for the closed/open system, see Grabmüller and Schädlich [7], did not give expressions for the first and second moments of RTD. It should be noted that their expression for $C(t)$, for long residence times, predicts a finite concentration at the outlet from the reactor. This finite concentration is equal to $\mathrm{Pe} /\left(1-\mathrm{e}^{-\mathrm{Pe}}\right)$. We feel that this solution cannot be correct because, in order to satisfy the mass balance, the limiting concentration should be equal to zero. Undoubtedly, this is caused by the incorrect boundary condition at $Z=1$, which states that $\mathrm{d} C / \mathrm{d} Z=C \mathrm{Pe}$ is always positive at the outlet from the reactor! 
Table 3. Types of boundary conditions and solutions of the PD-Model.

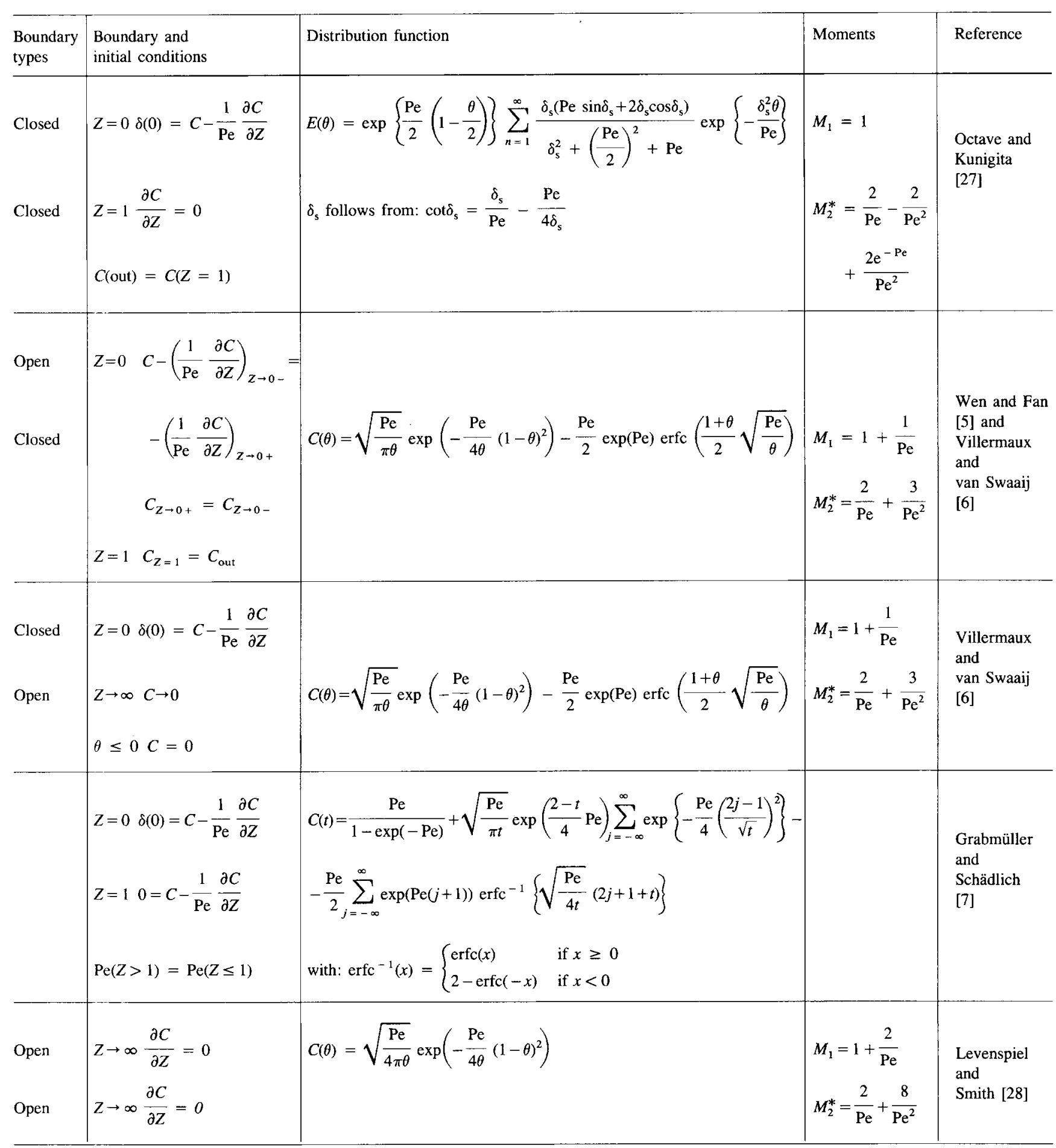

It can be seen from Table 3 that the value of the first moment is 1 only for a closed/closed system. For all other systems, this value differs from unity for reasons previously discussed.

\section{The Imperfect Pulse Method}

The imperfect pulse method was used to measure the system response to a tracer pulse input. According to this method, this response is measured at two locations in the system. The response curve at the first upstream detection point is taken as the imperfect pulse input for the system. The parameters can be calculated using the difference between the moments of both response curves.

The $k$ th moment of a distribution is defined as:

$M_{\mathrm{k}}=\int_{0}^{\infty} C(t) t^{k} \mathrm{~d} t$ 
Frequently, central moments, i.e. those around the mean, are used. These are defined as:

$M_{\mathrm{k}}^{*}=\int_{0}^{\infty} C(t)(t-\bar{t})^{k} \mathrm{~d} t$

in which $\bar{t}=M_{1}$. The second central moment $M_{2}^{*}$ is known as the variance of the distribution and can be used to calculate the dispersion coefficient.

For a system with open boundaries, the parameters for the PDModel can be calculated from:

$\bar{t}=\bar{t}_{\text {out }}-\bar{t}_{\text {in }}=M_{1, \text { out }}-M_{1, \text { in }}$

and

$\Delta \sigma^{2}=M_{2, \text { out }}^{*}-M_{2, \text { in }}^{*}=2 / \mathrm{Pe}$,

in which the first subscript of the moment indicates its order. The advantage of the imperfect pulse method is that the end effects are eliminated and that the actual bed response is measured, provided that neither injection nor detection devices introduce disturbances into the flow pattern. Especially in three phase reactors, which often possess a mixing chamber at the inlet and a separator at the outlet, this is a considerable advantage.

One of the difficulties encountered when using the method of moments is the phenomenon known as tailing: a weak signal continues for a very long time after the main part has passed the detection device. Tailing causes the higher moments to be unreliable; actually when tailing is observed one should already be very careful even when using the second moment. The reason is that, in the tail of the distribution, the measured values of tracer concentration are very small and, therefore, the relative errors at these points are large. For the $k$ th moment, the measured concentration is multiplied by $t^{k}$ which, at long times, becomes very large. This causes the higher moments to be unreliable, $t^{k}$ is in fact a weighting function by which all data points are multiplied. As follows from the already given explication, a better weighting can be achieved when the weighting function decreases with time. Such a weighting function is $\mathrm{e}^{-s t} t^{k}$; moments using this function are called weighted moments.

\section{The Weighted Moments Method}

The use of weighted moments for the analysis of residence time distribution data was first suggested by Østergaard and Michelsen [8]. Since then, several publications have followed concerning the mathematical background and application of this method to several mixing models, e.g. those of Midoux and Charpentier [9], Michelsen and Østergaard [10], Anderssen and White [11, 12], Pham and Keey [13], Hopkins, Sheppard and Eisenklam [14], Abbi and Gunn [15]. Michelsen and Østergard [10] showed several methods to evaluate $\mathrm{Pe}$ and $\tau$ from weighted moments using constant as well as variable values for $s$. The method we apply uses a constant value for $s$.
The weighted moments are defined as:

$W_{\mathrm{k}}=\int_{0}^{\infty} C(t) t^{k} \mathrm{e}^{-s t} \mathrm{~d} t$

The introduction of $\mathrm{e}^{-s t}$ into the definition of weighted moment means in fact that we apply a Laplace transformation to the concentration distribution $C(t) . W_{0}$ is the Laplace transform of $C(t)$.

By transforming the concentration distributions from the time to the Laplace domain, they can be easily related to the Laplace transform of the system transfer function through the convolution theorem: the output response can be calculated by convolution of the input response and the system transfer function:

$C_{\text {out }}(t)=\int_{0}^{t} C_{\text {in }}\left(t^{*}\right) \operatorname{TR}\left(t-t^{*}\right) \mathrm{d} t^{*}$,

where $t^{*}$ is the variable in the integration. The Laplace transform of both sides yields on rearrangement:

$\widehat{\mathrm{TR}}=\frac{\hat{C}_{\text {out }}}{\hat{C}_{\mathrm{in}}}=\frac{W_{0, \text { out }}}{W_{0, \text { in }}}$

Neglecting end effects, the transfer function for the PD-Model is:

$\operatorname{TR}(\theta)=\sqrt{\frac{\mathrm{Pe}}{4 \pi \theta^{3}}} \exp \left(-\frac{\mathrm{Pe}}{4 \theta}(1-\theta)^{2}\right)$.

The Laplace transform of the transfer function is:

$\widehat{\mathrm{TR}}=\exp \left[\frac{\mathrm{Pe}}{2}\left(1-\sqrt{1+\frac{4 s \tau}{\mathrm{Pe}}}\right)\right]$.

Because the PD-Model contains two parameters, two relationships between the transfer function and the response curves are necessary. These relationships were derived by Michelsen and Østergaard [10]:

$\mathrm{Pe}=\frac{U_{0}\left(U_{0}+2 s U_{1}\right)}{\left(U_{0}+s U_{1}\right)}$,
$\tau=\frac{-U_{0} U_{1}}{U_{0}+2 s U_{1}}$,

where

$U_{0}=\ln (\mathrm{TR})=\ln \left(\frac{W_{0, \text { out }}}{W_{0, \text { in }}}\right)$,

$U_{1}=\left(\frac{W_{1}}{W_{0}}\right)_{\text {out }}-\left(\frac{W_{1}}{W_{0}}\right)_{\text {in }}$.

With the introduction of $\mathrm{e}^{-s t}$ into the definition of the moments, the Laplace parameter $s$ was introduced as a new variable. 
As follows from Eq. (10), the value of the Laplace transform of the transfer function is determined by the dimensionless Peclet number and the dimensionless product $s \tau$. A suitable value for $s \tau$ has to be chosen. $s \tau$ exerts a large influence on the values of the estimated parameters and thus on the correctness of the estimate, as will be shown in a later section.

\section{Experimental Set-up and Procedure}

The experimental set-up is shown schematically in Fig. 1. The liquid feed is pumped into the reactor by a piston pump. The maximum flow rate which can be achieved is $30 \mathrm{ml} / \mathrm{min}$. Hydrogen is fed into the reactor cocurrently. The hydrogen flow is controlled by a mass-flow controller. The upstream pressure is kept constant at $1.4 \mathrm{MPa}$. The maximum gas flow rate is $5500 \mathrm{ml}$ hydrogen $/ \mathrm{min}$. System pressure was varied between 0.2 and $1.2 \mathrm{MPa}$.

Gas and liquid are separated at the top of the bed. The liquid is collected in a buffer vessel. The gas leaves the system through a back-pressure controller, which is used to control the pressure in the reactor.

The reactor consists of a stainless steel pipe with an inner diameter of $65 \mathrm{~mm}$. The bed length is $500 \mathrm{~mm}$. The packing consists of glass cylinders with an average diameter of $3.8 \mathrm{~mm}$ $(\sigma=0.2 \mathrm{~mm})$ and average length of $4.8 \mathrm{~mm}(\sigma=0.9 \mathrm{~mm})$.

Conductivity cells are placed at two locations in the bed. These cells consist of two parallel circular gauzes, $3.5 \mathrm{~mm}$ in mesh, $54 \mathrm{~mm}$ in diameter, at a distance of $5 \mathrm{~mm}$. The gauzes are mounted in a teflon ring holder as shown in Fig. 2. In order to reduce flow disturbances as much as possible, the space between the gauzes is filled with packing material. The distance between the two cells is $420 \mathrm{~mm}$. The cells are connected to an HP3497A Data Acquisition and Control Unit (DACU) which can be connected to either cell by switching a relay inside the DACU. The concentration-time curves for both cells can be obtained by alternately connecting the conductivity meter to either cell.

A tracer pulse is injected into the liquid feed stream by means of a high pressure tracer injector, shown schematically in

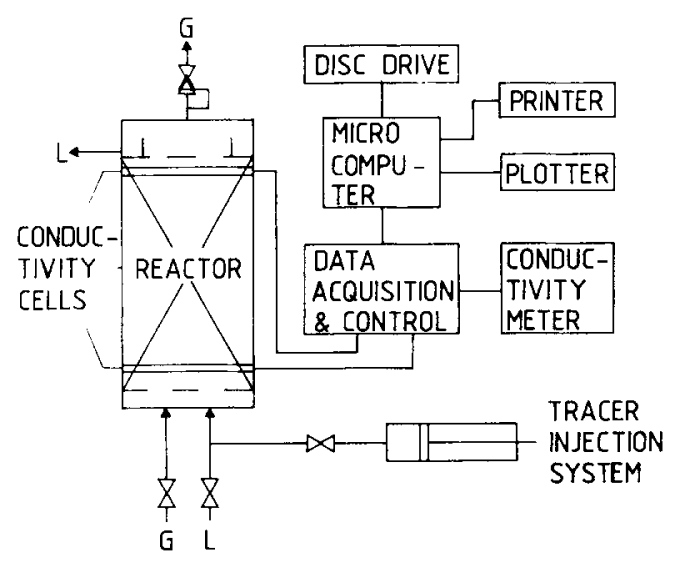

Fig. 1. Experimental set-up.

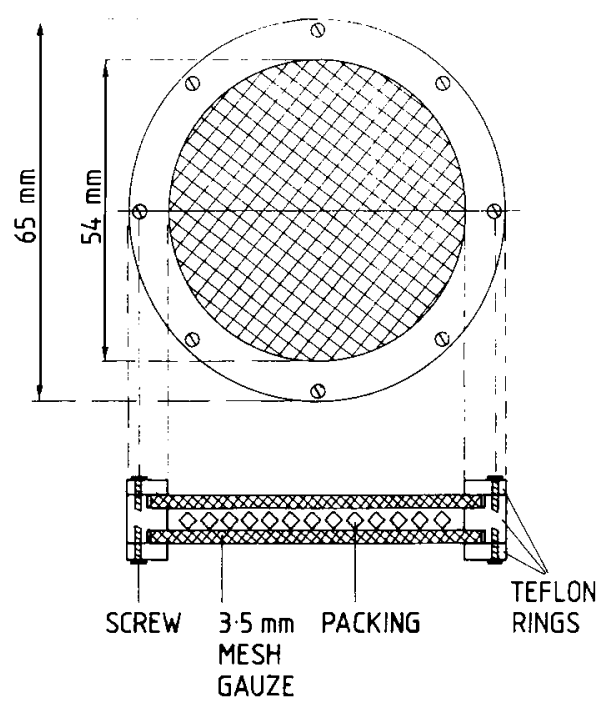

Fig. 2. Top and cross-sectional view of a conductivity cell.

Fig. 3. This injector consists of a primary piston with the diameter of $9.5 \mathrm{~mm}$. This primary piston is connected to a secondary piston, $2.1 \mathrm{~mm}$ in diameter. Because of the large difference in surface areas of the two pistons, low pressure air at 3 bar can be used to inject tracer solution into the feed stream. The stroke adjustor can be used to control the length of the stroke and thereby the injected tracer volume. $3.4 \mathrm{ml}$ of 0.054 $\mathrm{M} \mathrm{LiCl}$ solution in methanol is used as tracer.

The entire experiment is carried out automatically and controlled by a microcomputer except for the start-up and tracer injection. After each experiment, the measured data are stored on disk for further analysis. With this automation, 300 to 650 data points can be taken per curve.

Before the start of an experiment, the baselines are measured and checked for drift. The experiment is ended when the measured response in the second cell, i.e. the measured signal minus the baseline correction, is less then $1 \%$ of the maximum response for 10 consecutive data points. Each datum point is itself an average of five readings taken at $0.1 \mathrm{~s}$ intervals to com-

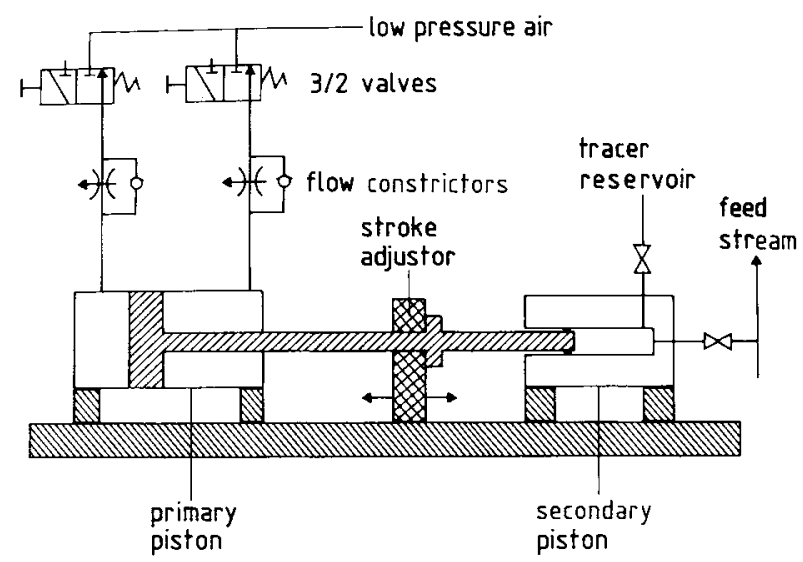

Fig. 3. High pressure tracer injector. 
pensate for small variations in the measured conductivity values caused by gas flow fluctuations.

\section{Influence of the Weighting Factor}

Before further analysis, the data were normalized. Since the experimental curves were already quite smooth, no further smoothing was necessary.

In order to calculate the parameters Pe and $\tau$ according to Eq. (11), a value for $s$ has to be chosen. Hopkins et al. [14] showed that not $s$ but the dimensionless product of $s$ and $\tau$ determines the correct choice of $s$. They also showed that if $s \tau$ is too small the effect of tailing is not completely eliminated because $\mathrm{e}^{-s t}$ does not decrease fast enough. On the other hand, if $s \tau$ is too large, tailing is completely eliminated but, at the same time, too much weight is given to the early values of the curves which are also small and therefore contain a large relative error. An optimum value for $s \tau$ must therefore exist. The optimum weighting factor depends on the shape of the response curves. The influence of $s$ was determined experimentally. First, $\tau$ was estimated from the difference between the first moments of output and input. With this estimate, $\tau^{*}$ values for $s$ were chosen so that $s \tau^{*}$ was varied in small steps of between 0.2 and 8.0. For each value of $s$, the parameters Pe and $\tau$ were calculated. With these parameters, the theoretical output response curve was calculated by convolution of the input response curve and the transfer function. Then, the difference area between the theoretical and experimental output response curves defined by:

$\Delta A=\int_{0}^{\infty}\left|C_{\mathrm{out}}^{\mathrm{exp}}(t)-C_{\mathrm{out}}^{\mathrm{theor}}(t)\right| \mathrm{d} t$

was calculated. In Fig. 4a $\tau(s \tau), \operatorname{Pe}(s \tau)$ and $\Delta A(s \tau)$ are plotted for one of the experiments. It is clear that there is an optimum value of $s \tau$ for which $\Delta A$ has a minimum value. The same plots were obtained for many experiments and they all showed a rather strong dependence of $\Delta A$ on $s \tau$. A clear minimum, such as in Fig. 4a, was not always found. Occasionally, the minimum was very flat or there were two minima separated by a local maximum (Fig. 4b). A minimum for $\Delta A$ was always obtained, but turning points for $\mathrm{Pe}(s \tau)$ and $\tau(s \tau)$ were not always found. It can be proven that the sign of $\mathrm{dPe} / \mathrm{d} s$ is always opposite to that of $\mathrm{d} \tau / \mathrm{d} s$. It can also be proven that if $\tau(s)$ has an extreme
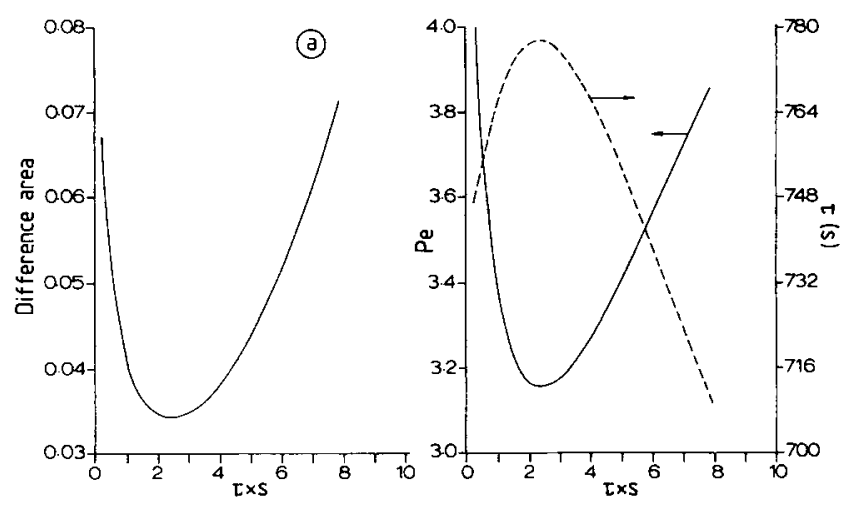
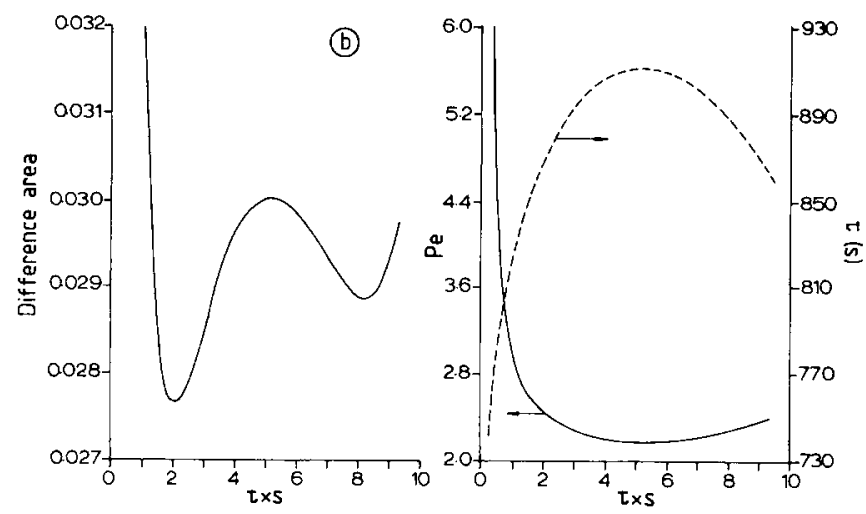

Fig. 4. Influence of product $s \tau$ on calculated parameters.

for a value of $s$, then Pe and $\Delta A$ also have extremes for the same value of $s$. However, for $\Delta A$ this point may be a minimum (Fig. 4a) as well as a (local) maximum as in Fig. 4b. It is therefore not possible to find the minimum of $\Delta A$, and thus the optimum weighting factor, by finding the extremes of $\tau(s)$ or $\operatorname{Pe}(s)$.

The optimum value for $s \tau$ varied between 0.5 and 2.5. As seen from Fig. $4 \mathrm{a}$, an incorrect choice of $s \tau$ can have a dramatic effect on the accuracy of the calculated parameters. Therefore, it was necessary to calculate the optimum $s \tau$ for each experiment separately.

\section{Parameter Evaluation}

In view of the results discussed in the previous paragraph, the following procedure was adopted for the evaluation of the parameters:

- A value for $s$ was estimated using an equation of Anderssen and White [11]

$s=\frac{2\left(k_{\mathrm{av}}+1\right)}{\bar{t}_{\mathrm{out}}+\bar{t}_{\mathrm{in}}-\Delta t_{D}}$,

in which $k_{\mathrm{av}}$ is the average order of the moments used to estimate the parameters and $\Delta t_{\mathrm{D}}$ the time delay between the input and output signals.

- With this value for $s$, a first estimate of $\tau$ was calculated: $\tau^{*}$

- Now $s r^{*}$ was varied between 0.4 and 4.0 in steps of 0.3 , i.e. $s$ was varied between $0.4 / \tau^{*}$ and $4.0 / \tau^{*}$ in steps of $0.3 / \tau^{*}$.

- For each value of $s$, the parameters Pe and $\tau$ were calculated.

- With these parameters, the theoretical output response was calculated by convolution and then the difference area was computed.

- The values of Pe and $\tau$, resulting in the smallest difference area, are taken as the best estimate of these parameters. Since convolution of a curve consisting of $N$ points requires $N+(N-1)+(N-2)+\ldots+2+1=(N / 2) *(N+1)$ evaluations of the transfer function, this procedure was very time consuming; however, it was necessary for the previously discussed reasons. 


\section{Experimental Results}

The results of model calculations are the values of the Peclet number and of the average residence time. Peclet number is based on the distance between the detection probes and on the true liquid velocity $U_{\mathrm{L}} / \varepsilon_{\mathrm{L}}$ while $\tau$ is based on the true liquid volume between the detection probes. For the interpretation of results, it is better to use the Bodenstein number and the liquid hold-up. The Bodenstein number is defined as $\mathrm{B}_{0}=U_{\mathrm{LS}} d_{\mathrm{eq}} /\left(\varepsilon_{\mathrm{L}} D_{\mathrm{ax}}\right)=$ Pe $d_{\text {eq }} / L$ and depends on the equivalent packing diameter and not on reactor length. The equivalent spherical diameter is used as the characteristic packing diameter which, for a cylinder with the same external surface as a sphere with diameter $d_{\text {eq }}$, is given by the equation:

$d_{\mathrm{eq}}=\sqrt{\frac{d_{\mathrm{p}}^{2}}{2}+d_{\mathrm{p}} L_{\mathrm{p}}}$.

The ratio $L / d_{\text {eq }}$ is equal to 83.5 for the distance between the detection probes and our packing. In this paper, the hold-up $\varepsilon$ is defined as the fraction of the reactor volume occupied by a phase, thus $\varepsilon_{\mathrm{L}}+\varepsilon_{\mathrm{G}}+\varepsilon_{\mathrm{S}}=1$.

\subsection{Zero Gas Flow Rate}

Two experiments were conducted with no gas flowing through the reactor. Typical response curves are shown in Fig. 5. The liquid phase Reynolds numbers for these experiments were 0.99 and 0.58 . The calculated values for Bo were 0.52 and 0.58 . These values are in good agreement with literature data for single phase flow in packed beds at low Reynolds numbers, see Westerterp et al. [16], p. 213. The calculated values for $\tau$ were $1050 \mathrm{~s}$ and $1870 \mathrm{~s}$, both resulting in a value of 0.365 for the hold-up. This value is equal to the void fraction of the bed because there is no gas flowing through it.

\subsection{Three-phase Operation}

Approximately 100 experiments were carried out with gas and

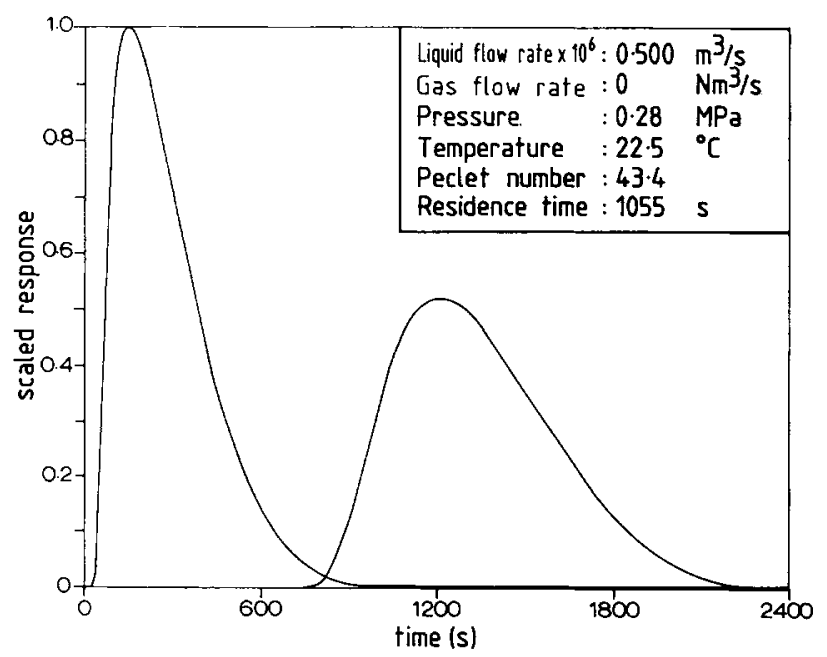

Fig. 5. Response curves for zero gas flow rate. liquid flowing through the reactor simultaneously. Gas and liquid flow rate and reactor pressure were varied. Typical response curves are shown in Fig. 6. Table 4 gives a complete list of experimental conditions and calculated results.

In Fig. 7a, the calculated Bodenstein number is plotted as a function of the gas phase Reynolds number and, in Fig. 7b, as a function of superficial gas velocity at reactor pressure. In both diagrams, parameter is the liquid feed rate. It follows from Fig. 7a that there is practically no correlation between Bo and $\mathrm{Re}_{\mathrm{G}}$; the only conclusion which can be drawn from this diagram is that Bo increases when liquid feed rate increases. The influence of superficial gas velocity at reactor conditions as given in Fig. $7 \mathrm{~b}$ is much more pronounced; Bo decreases with increasing gas velocity.

In Fig. 8a, the liquid phase hold-up is plotted as a function of the gas phase Reynolds number and, in Fig. $8 \mathrm{~b}$, as a function of the superficial gas velocity at reactor conditions. Again, there is no apparent correlation between $\varepsilon_{\mathrm{L}}$ and $\operatorname{Re}_{\mathrm{G}}$ but the influence of the gas velocity is clear: the liquid hold-up decreases with increasing gas velocity.

\subsection{Accuracy of the Calculated Parameters}

The accuracy of the calculated parameters is described by the difference area as defined in Eq. (13). One should bear in mind that the area under both the experimental and the theoretical output response curve is equal to 1 . The average value of the difference area is 0.024 with a standard deviation of 0.008 . This value compares very favourably with the values found by Kan and Greenfield [17] for their three parameter model. They found an average value for $\Delta A$ of approximately 0.10 .

Our very low $\Delta A$ values lead to the conclusion that the plug flow with axial dispersion model describes mixing in our system adequately. Therefore, it is not necessary to use a model with more parameters.

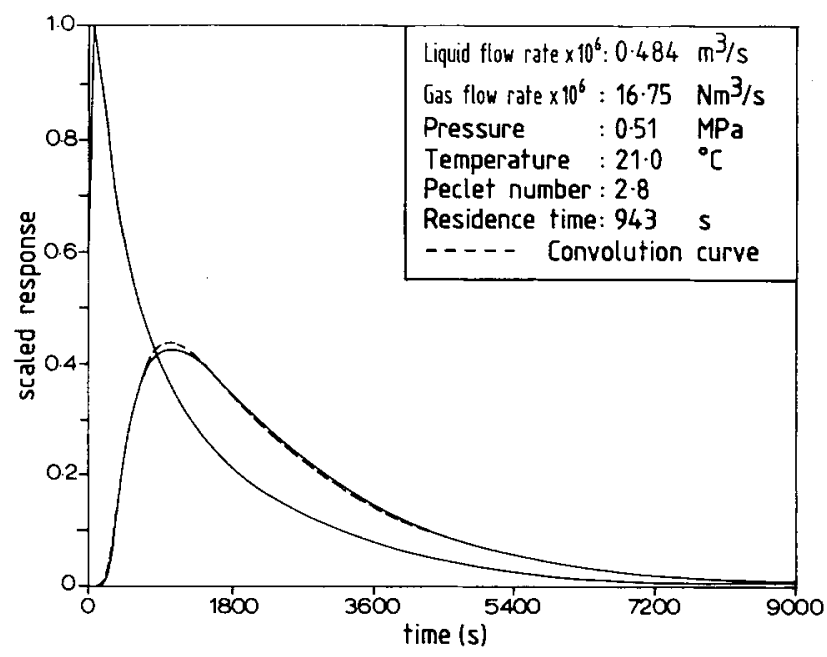

Fig. 6. Response curves for three-phase operation. 
Table 4. Experimental conditions and calculated results.

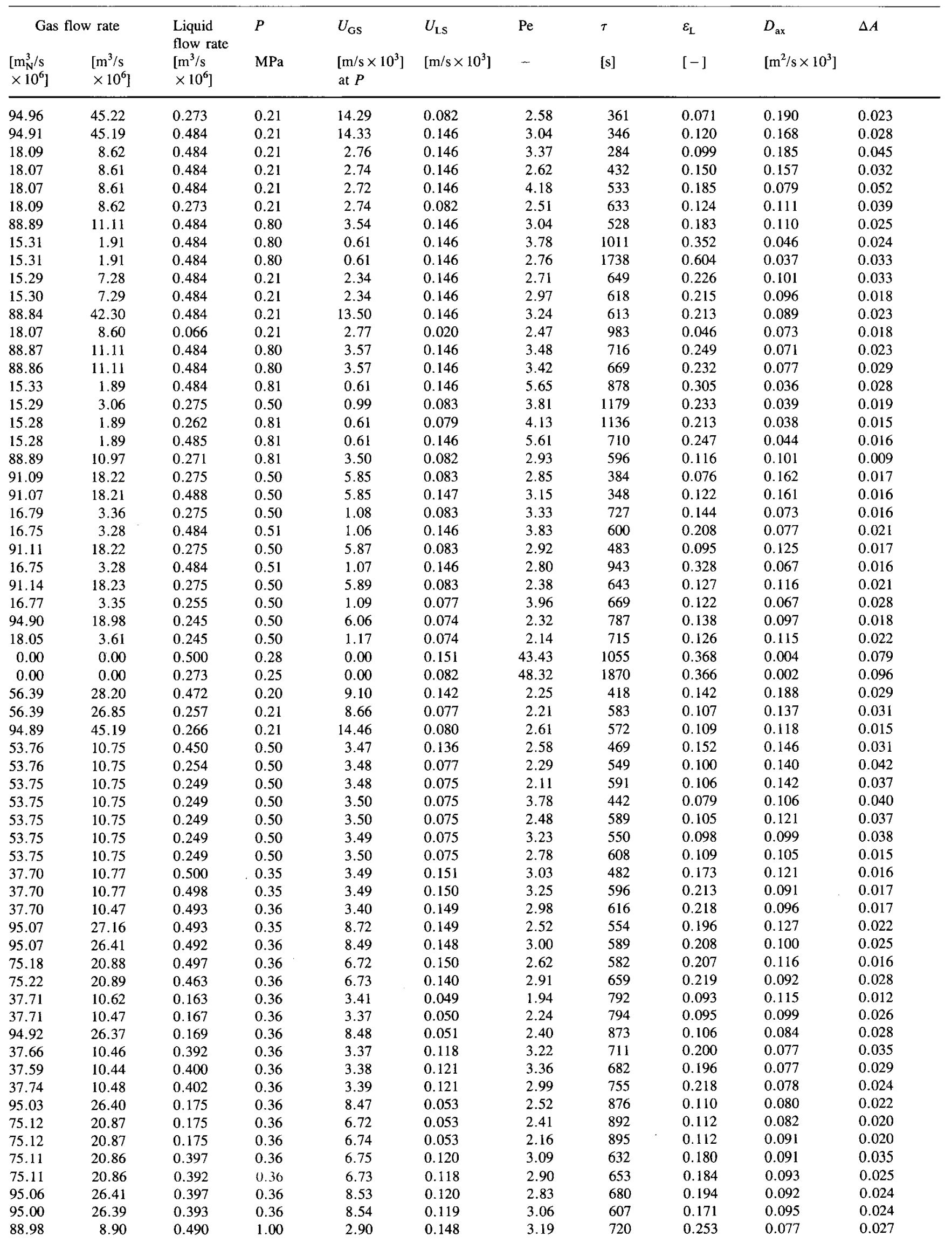


Table 4. Continued

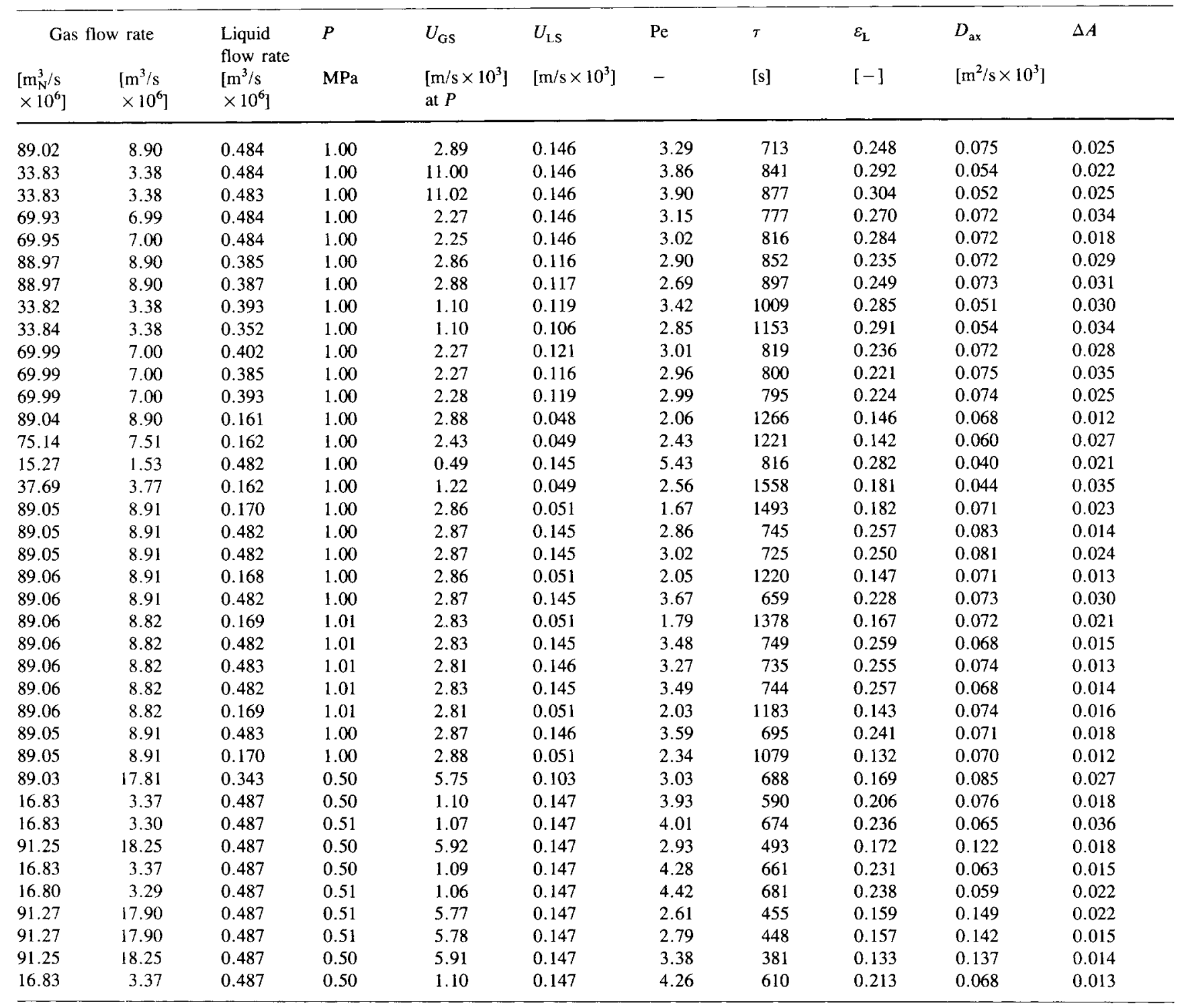

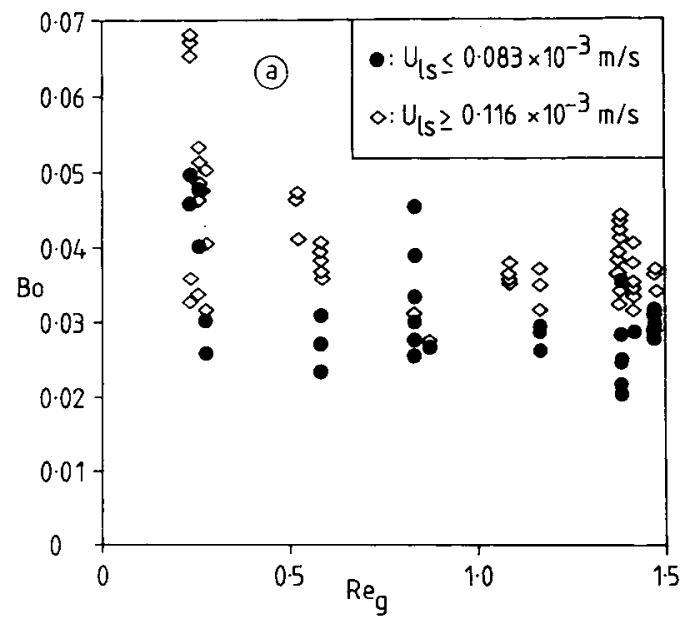

Fig. 7. Bodenstein number as a function of gas and liquid loading of column; a) Bodenstein number versus gas phase Reynolds number;

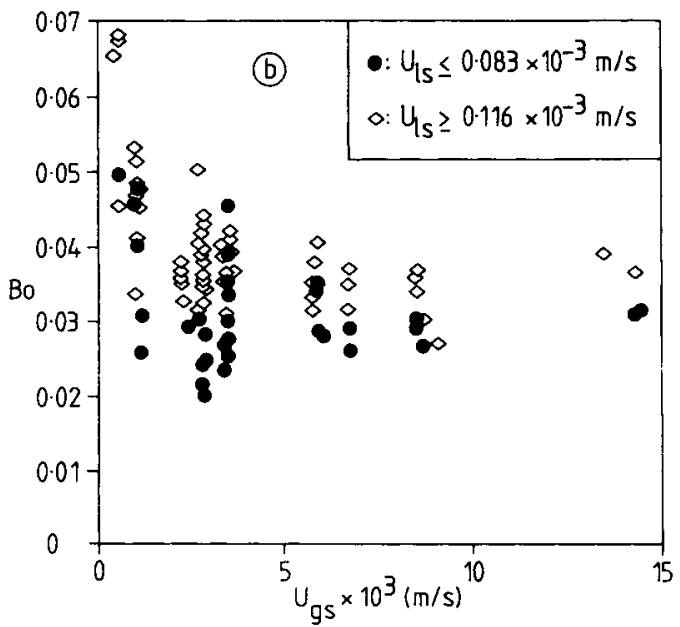

b) Bodenstein number versus superficial gas velocity at reactor conditions. 

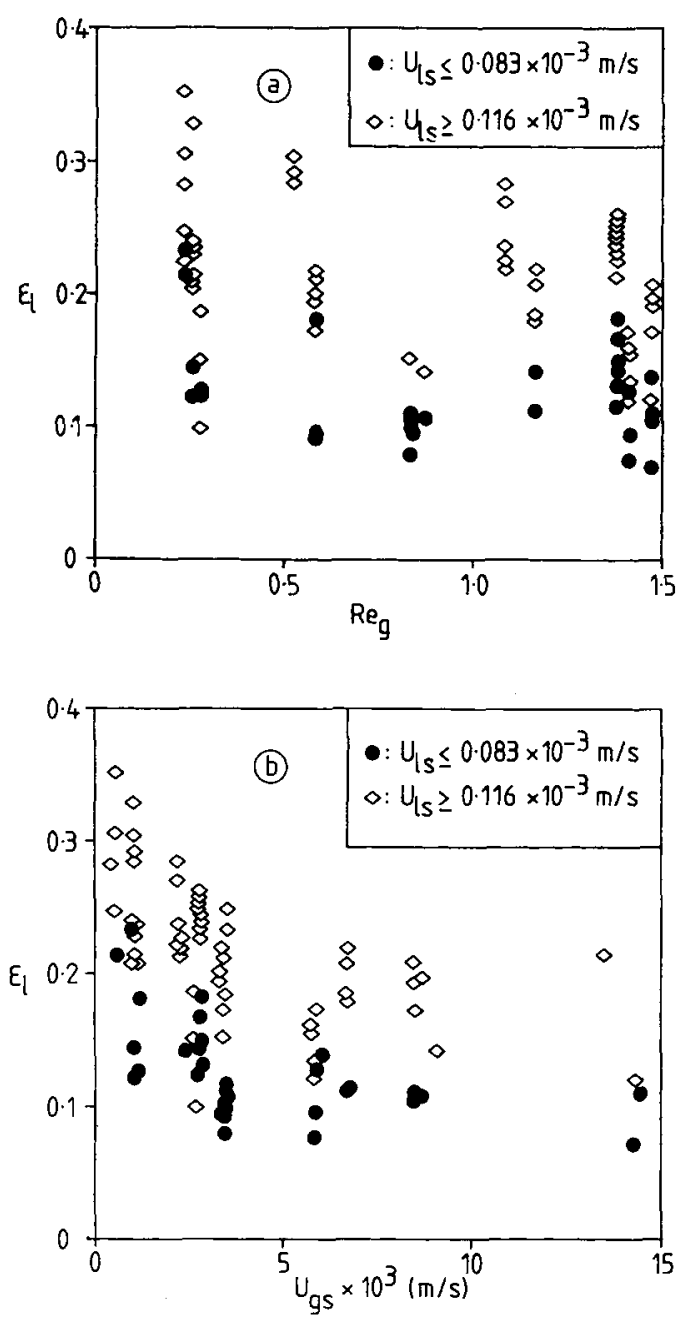

Fig. 8. Liquid phase hold-up as a function of gas liquid loading of column; a) $\varepsilon_{\mathrm{L}}$ versus gas phase Reynolds number; b) $\varepsilon_{\mathrm{L}}$ versus superficial gas velocity at reactor conditions.

\subsection{Comparison Between the Use of Weigthed and Normal Moments}

The method of weighted moments which we use to calculate the parameters requires a vast computational effort. To verify whether this effort is worthwhile, our results should be compared to those obtained by the method of ordinary moments. Table 5 presents such a comparison between the ordinary moments method (OMM) as described by Eqs (4) and (5) and the weighted moments method (WMM) as modified by us. It is clear from this table that the estimation of the parameters with the WMM is up to 5 times more accurate. With the OMM, the average residence time can be estimated with reasonable accuracy; however, the estimated Peclet number is far from accurate. We believe that this is caused by the observed tailing in the response curves. Especially for reactor calculations involving more complex reaction schemes, the difference between a Peclet number of, for example, 2 and 3 can be very significant. We must therefore conclude that the results obtained with the Weigthed Moments Method justify the extra computational effort needed.
Table 5. Comparison of results obtained with the weighted moments method (WMM) as modified by the authors and with the ordinary moments method (OMM).

\begin{tabular}{rc|cc|cc}
\hline & $\begin{array}{c}\tau \\
{[\mathrm{s}]}\end{array}$ & \multicolumn{2}{c|}{$\begin{array}{c}\text { Pe } \\
{[-]}\end{array}$} & \multicolumn{2}{c}{$\begin{array}{c}\Delta A \\
{[-]}\end{array}$} \\
\hline OMM & WMM & OMM & WMM & OMM & WMM \\
\hline 1025 & 1195 & 5.9 & 2.1 & 0.097 & 0.016 \\
630 & 660 & 2.7 & 3.7 & 0.056 & 0.031 \\
680 & 695 & 2.5 & 3.6 & 0.089 & 0.021 \\
1110 & 1090 & 5.2 & 2.3 & 0.068 & 0.012 \\
365 & 380 & 6.8 & 3.4 & 0.070 & 0.015 \\
610 & 610 & 3.3 & 4.3 & 0.047 & 0.013 \\
630 & 675 & 4.0 & 4.0 & 0.074 & 0.038 \\
490 & 495 & 3.9 & 3.0 & 0.037 & 0.020 \\
\hline
\end{tabular}

\section{Correlation and Discussion}

Different correlations with either Reynolds numbers or superficial velocities at reactor conditions and based on the empty column diameter were tested to correlate the calculated parameters. Of these parameters only the superficial gas velocity at constant mass flow rate is influenced by reactor pressure.

\subsection{Axial Dispersion}

The different types of correlations tested for Bo are given in Table 6. In these correlations, $X$ is the parameter for the liquid phase $\left(\operatorname{Re}_{\mathrm{L}}\right.$ or $\left.U_{\mathrm{LS}}\right)$ and $Y$ the parameter for the gas phase $\left(\operatorname{Re}_{\mathrm{G}}\right.$ or $U_{\mathrm{GS}}$ ). The constant 0.5 follows from the consideration that, at low Reynolds numbers, $\mathrm{Bo}=0.5$ for packed beds and liquid flow only.

For every correlation, the parameters resulting in the best fit for Bo were calculated by means of a non-linear least squares procedure based on the Marquardt's method. This resulted in 34 different equations for Bo. From these, the best correlation had

Table 6. Correlations tested for the Bodenstein number.

\begin{tabular}{ll}
\hline$a+b X^{c}+d Y^{e}$ & $0.5+b X^{c}+d Y^{e}$ \\
$a+b X^{c}+d(Y / X)^{e}$ & $0.5+b X^{c}+d(Y / X)^{e}$ \\
$a+b X^{c}$ & $0.5+b X^{c}$ \\
$a+d Y^{e}$ & $0.5+d Y^{e}$ \\
$a+b(Y / X)^{e}$ & $0.5+b(Y / X)^{e}$ \\
$a+b X^{c} Y^{e}$ & $0.5+b X^{c} Y^{e}$ \\
$b X^{c}+d Y^{e}$ & \\
$b X^{c} Y^{e}$ & \\
$b(Y / X)^{e}$ & \\
$b X^{c}$ & $d Y^{e}$
\end{tabular}

Note: $X$ denotes the parameter for the liquid phase, i.e. $\mathrm{Re}_{\mathrm{G}}$ or $U_{\mathrm{LS}}, Y$ denotes the parameter for the gas phase, i.e. $\operatorname{Re}_{\mathrm{L}}$ or $U_{\mathrm{GS}}$. 
to be selected. As a first selection criterion, the standard error as defined by the following equation was used:

standard error $=\sqrt{\frac{\text { SSR }}{\left(\begin{array}{l}\text { Number } \\ \text { of data }\end{array}\right)-\left(\begin{array}{l}\text { Number of } \\ \text { parameters }\end{array}\right)}}$,

in which SSR is the sum of squared residuals.

Table 7 lists the best correlations, arranged in the order of increasing standard errors. It is clear from this table that correlations based on superficial velocity at reactor conditions give the best results. The last 5 columns give the values and the $95 \%$ confidence interval for the calculated parameters. The confidence interval is expressed as the percentage of the absolute value of the parameter. Correlations for which one or more confidence intervals are larger than $100 \%$ are unsatisfactory because the parameter(s) involved are not distinctly positive or negative; these correlations were not included in Table 7. For several correlations, the calculation of the parameters did not converge. Such correlations were also excluded from the selection.

Because we did not vary the viscosity of the gas phase (gas viscosity is practically constant at the relatively low pressures applied) nor the viscosity or density of the liquid phase, we prefer to use correlations which are not based on Reynolds numbers. Therefore, for this system, we propose to use the correlation $7-1$ for the calculation of $\mathrm{Bo}$ :

$\mathrm{Bo}=0.065 U_{\mathrm{LS}}^{0.310} U_{\mathrm{GS}}^{-0.177}$.

A parity plot of the observed Bodenstein number versus that calculated with this equation is shown in Fig. 9. The broken lines in this diagram give the $20 \%$ deviation range from the calculated value. As follows from Fig. 9, there is a considerable scatter of data. Such scatter was also observed by several other workers; Hochman and Effron [18], Saez and Carbonelli [19], Sater and Levenspiel [20], Stiegel and Shah [21], Kan and Greenfield [17], Turpin and Huntington [22]. Apparently, there are significant random variations in the dispersion. These variations can be caused by the random character of the dispersion or by small fluctuations in, for instance, feed temperature or feed rate. Apparently, it is not possible to determine the Bodenstein number with an error smaller than approximately $20 \%$.

Except for correlation 7-6, no comparison can be made with correlations from literature. In 1977, Stiegel and Shah [21] proposed a correlation of the same form, see Table 2 . The agreement between their coefficients for $\operatorname{Re}_{L}(0.245$ versus 0.31$)$ and $\operatorname{Re}_{G}(0.16$ versus 0.177$)$ and ours is remarkable. Stiegel and Shah used water and air (in contrast to methanol/hydrogen), worked at atmospheric pressure and applied Reynolds numbers up to 100 times larger than ours. This may point to the possibili-

Table 7. Bo correlations in order of ascending standard errors.

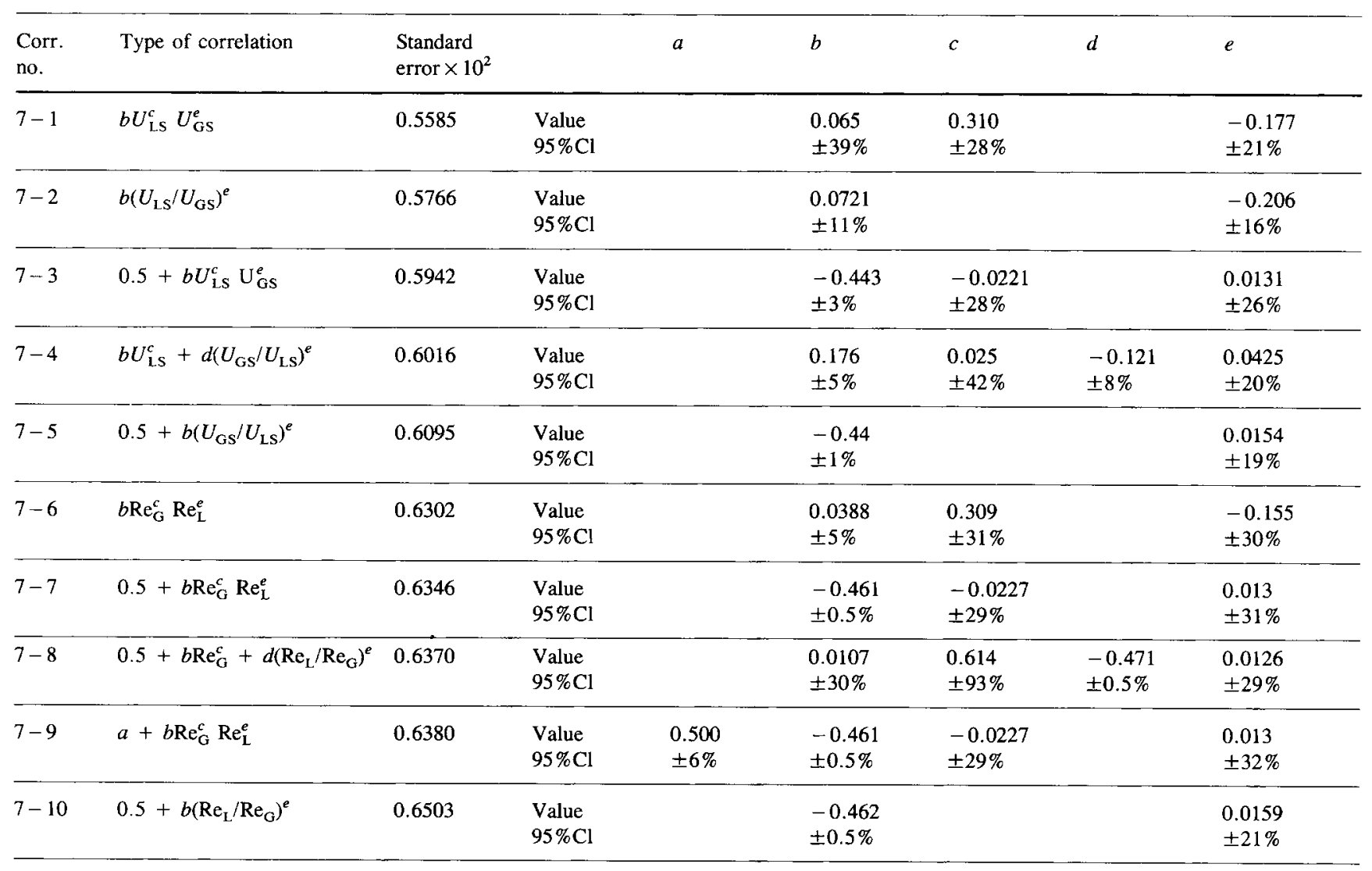




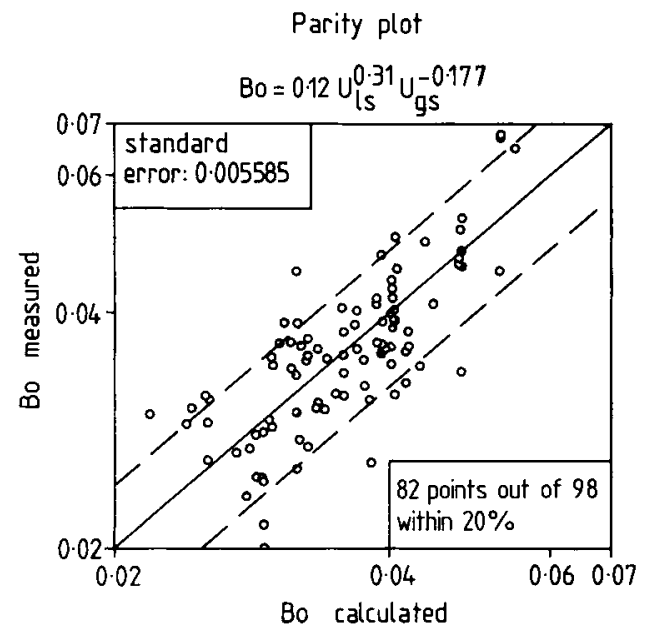

Fig. 9. Parity plot of proposed correlation for Bodenstein number.

ty of a general correlation for Bo for widely differing systems, covering a wide range of liquid and gas loadings, if right correlations can be found for system properties.

\subsection{Hold-up}

The same types of correlations as those adopted for the Bodenstein number were used for correlating the hold-up, see Table 6.
The constant in the right hand column of Table 6 was altered from 0.5 to 0.365 , the bed void fraction. Again, the parameters resulting in the best fit for $\varepsilon_{\mathrm{L}}$ were calculated and the correlations are arranged in the ascending order of standard errors. The best correlations are given in Table 8 . The 5 columns on the right again represent the calculated values of the parameters and the $95 \%$ confidence intervals as percentages of the parameter values. Again, correlations for which one or more confidence intervals exceeded $100 \%$ were not included in the selection. Non-convergent correlations were also excluded. It is apparent that, again, the correlations based on superficial velocity at reactor conditions yield the best results.

For the calculation of liquid hold-up, we propose the use of correlation 8-2 because of the small confidence intervals for the calculated parameters:

$\varepsilon_{\mathrm{L}}=0.354+0.143 U_{\mathrm{LS}}^{0.405}-0.206\left(U_{\mathrm{GS}} / U_{\mathrm{LS}}\right)^{0.125}$

On account of its simplicity, the use of correlation $8-1$ could also have been considered:

$\varepsilon_{\mathrm{L}}=0.341 U_{\mathrm{LS}}^{0.583} U_{\mathrm{GS}}^{-0.199}$

Fig. 10 presents a parity plot of the observed values for $\varepsilon_{\mathbf{L}}$ versus those calculated by Eq. (18). It is clear that there is much more scatter in the hold-up data than in the dispersion data. This can be caused by minor variations in the gas and liquid flow

Table 8. Hold-up correlations in order of ascending standard errors.

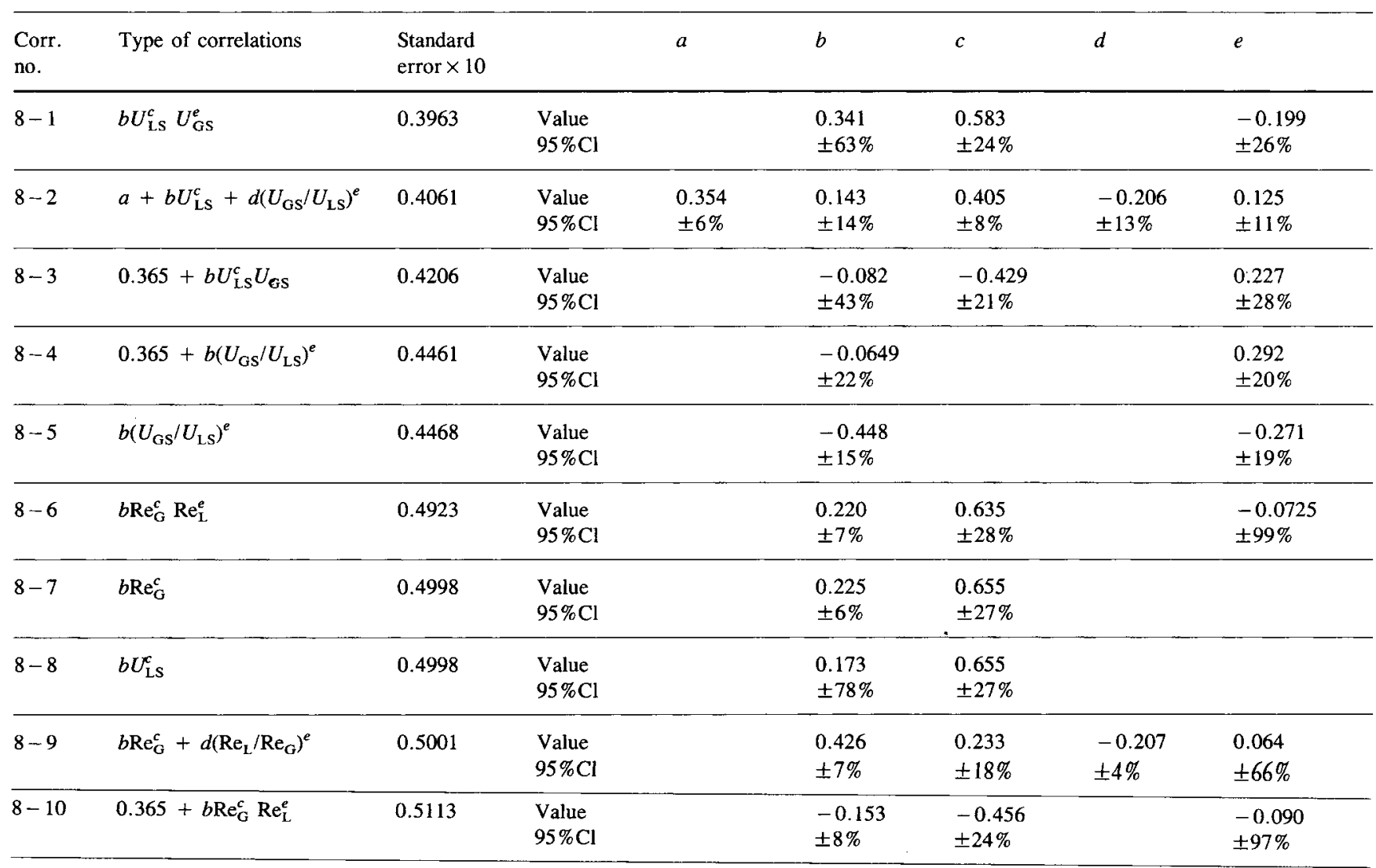




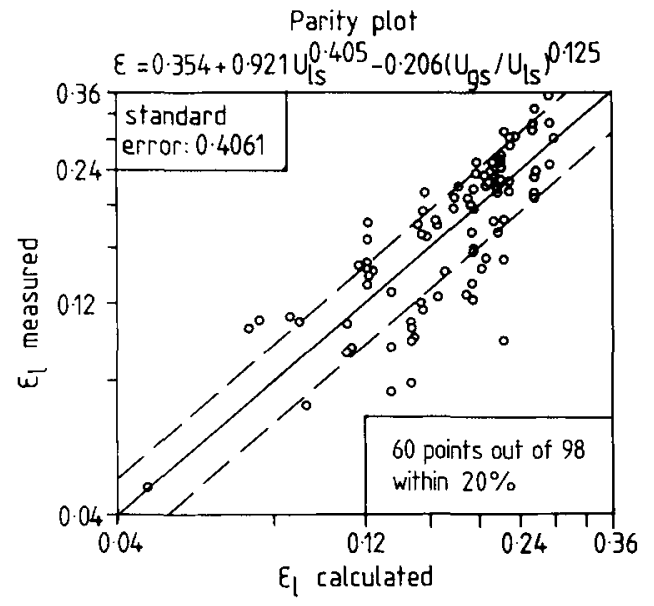

Fig. 10. Parity plot of proposed correlation for hold-up.

rates during the experiments. Comparing Eqs (17) and (19), it is clear that $\varepsilon_{\mathrm{L}}$ depends more strongly on gas and liquid flow rates than Bo. Consequently, variations in the flow rates will have a greater effect on $\varepsilon_{\mathrm{L}}$ than on Bo.

We can compare the correlation $8-6$ with that for the hold-up, given by Stiegel and Shah, see Table 1. Although they defined the hold-up on the basis of the bed void fraction, we can still compare the coefficients for $\operatorname{Re}_{\mathrm{L}}$ and $\mathrm{Re}_{\mathrm{G}}$. We find a much stronger dependence of $\varepsilon_{\mathrm{L}}$ on the liquid phase Reynolds number. This discrepancy may be attributed to the large differences between the flow rates of our experiments and theirs.

\subsection{Axial Dispersion Coefficient}

From the values calculated for Bo and $\varepsilon_{\mathrm{L}}$ it is possible to calculate those for the axial dispersion coefficient $D_{\text {ax }}$ :

$D_{\mathrm{ax}}=\frac{U_{\mathrm{LS}} d_{\mathrm{eq}}}{\varepsilon_{\mathrm{l}} \mathrm{Bo}}$

The correlations of Table 6 were used to express $D_{\mathrm{ax}}$ as a function of the velocities ( $U_{\mathrm{LS}}$ and $U_{\mathrm{GS}}$ ) or the Reynolds numbers $\left(\operatorname{Re}_{\mathrm{L}}\right.$ and $\left.\operatorname{Re}_{\mathrm{G}}\right)$. The coefficients for $U_{\mathrm{LS}}$ or $\mathrm{Re}_{\mathrm{L}}$ showed very large confidence intervals. A statistical analysis of the data showed that there is practically no correlation between $D_{\mathrm{ax}}$ and $U_{\mathrm{LS}}$ or $\mathrm{Re}_{\mathrm{L}}$. This is not surprising since the magnitude of $D_{\mathrm{ax}}$ for single phase liquid flow is of the order of $10^{-6} \mathrm{~m}^{2} / \mathrm{s}$ whereas for gas-liquid flow it is of the order of $10^{-4} \mathrm{~m}^{2} / \mathrm{s}$. Thus, it can be understood that the axial dispersion coefficient is almost exclusively influenced by the gas flow rate and that the influence of the liquid flow rate is practically negligible. A satisfactory correlation of $D_{\mathrm{ax}}$ is found with the linear gas velocity resulting in:

$D_{\mathrm{ax}}=a U_{\mathrm{GS}}^{b}$,

in which

$a=0.308 \times 10^{4}$ with a $95 \% \mathrm{Cl}$ of $9 \%$,

$b=0.313$ with a $95 \% \mathrm{Cl}$ of $25 \%$.
A parity plot of this correlation is shown in Fig. 11. It is clear that the scatter of data is very extensive. The reason is that the calculated values of $D_{\mathrm{ax}}$ contain accumulated errors both in the values of $B o$ and of $\varepsilon_{L}$.

\section{Conclusions}

The WMM is more satisfactory than the OMM in the estimation of parameters for the plug flow with dispersion model. However, it is important to use a correct weighting factor for the WMM. Finding the optimum weighting factor requires a large computational effort, but produces very good estimates of the model parameters Pe and $\tau$, resulting in a very small difference area between the theoretical and experimental response curves. The average value for $\Delta A$ was 0.024 .

Although the parameters for each individual experiment could be estimated with a high degree of accuracy, the reproducibility of experiments was poor.

The best correlations for Bo and $\varepsilon_{\mathrm{L}}$ were obtained when the superficial velocities for the phases at reactor conditions were used. This implies that the reactor pressure influences both Bo and $\varepsilon_{\mathrm{L}}$. The effect of pressure on the values of Bo and $\varepsilon_{\mathrm{L}}$ can be fully accounted for by using the linear gas velocity at the system pressure. Eq. (17) is proposed for Bo and Eq. (18) for $\varepsilon_{\mathrm{L}}$. It was concluded that the axial dispersion coefficient depends only on gas velocity, again at reactor conditions.

Received: March 2, 1989 [CET 212]

\section{Symbols used}

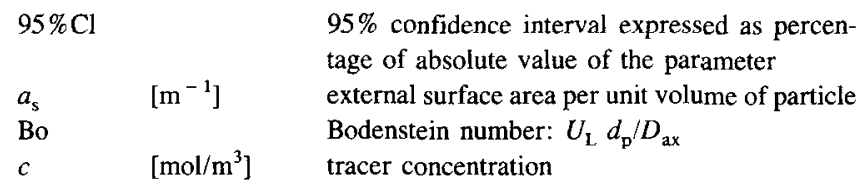

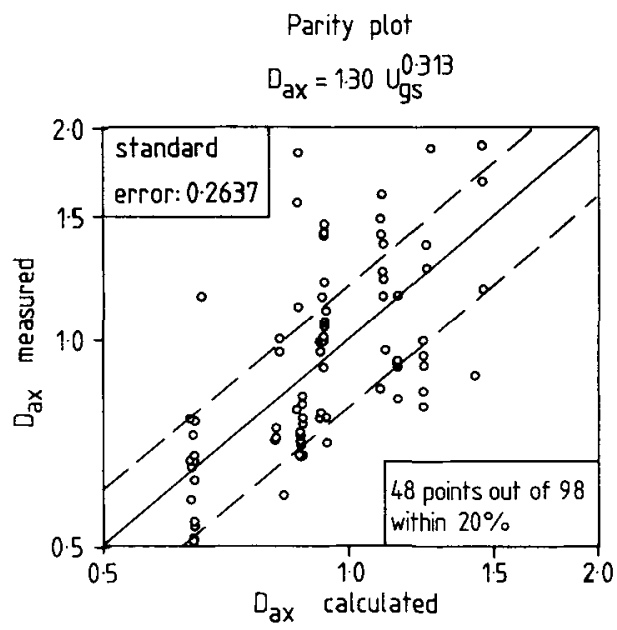

Fig. 11. Parity plot of correlation for axial dispersion coefficient. 


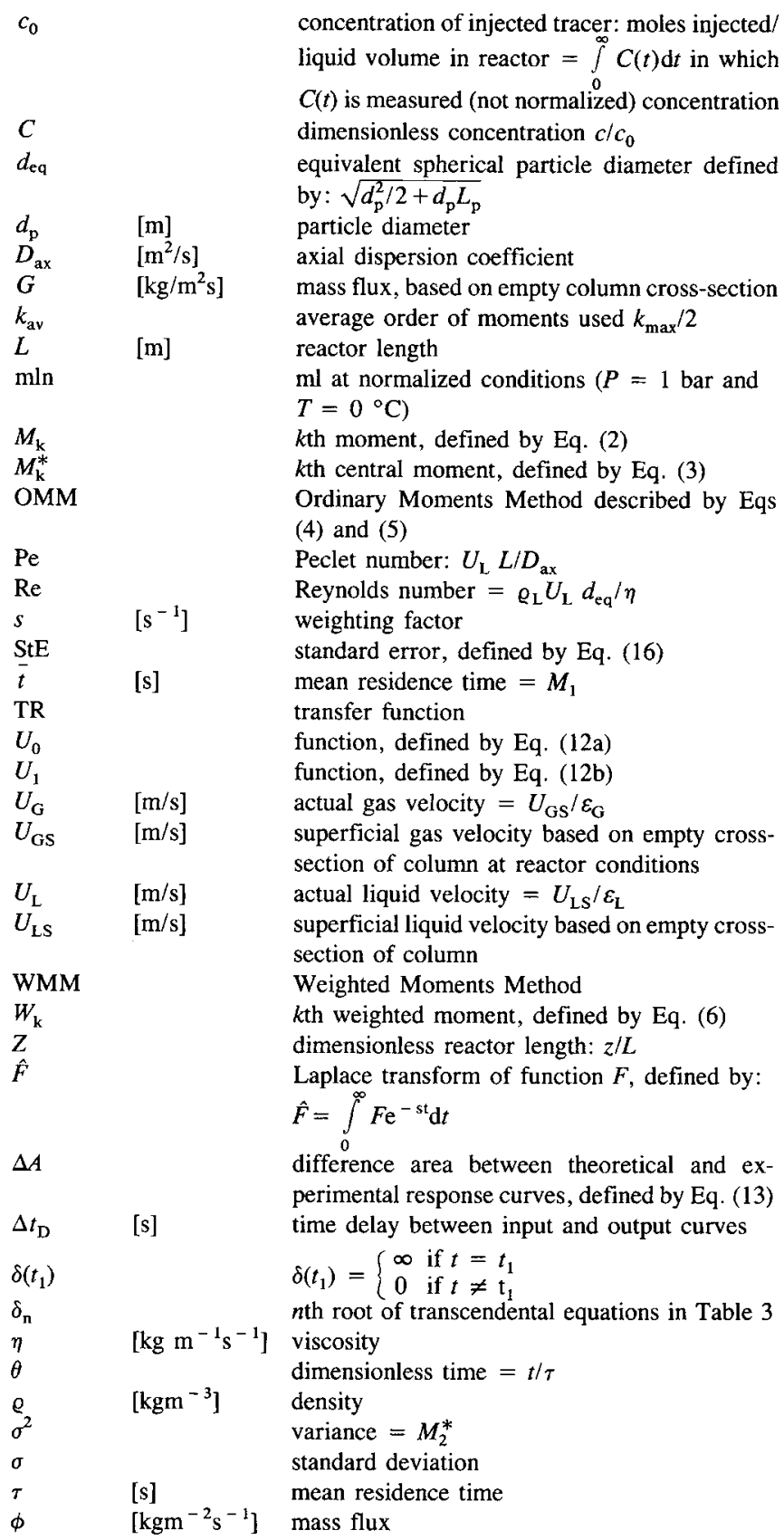

Indices

$\begin{array}{ll}\text { av } & \text { average } \\ \text { ax } & \text { in axial direction } \\ \text { calc } & \text { calculated } \\ \text { exp } & \text { experimental } \\ \text { G } & \text { gas phase } \\ \text { in } & \text { input } \\ \text { L } & \text { liquid phase }\end{array}$

\begin{tabular}{|c|c|}
\hline $\mathrm{m}$ & mass \\
\hline out & output \\
\hline $\mathrm{p}$ & particle \\
\hline$s$ & $\begin{array}{l}\text { superficial, based on empty cross-section of column at reactor } \\
\text { conditions }\end{array}$ \\
\hline theor & theoretical \\
\hline
\end{tabular}

\section{References}

[1] Shah, Y.T., Gas-solid-liquid reactor design, McGraw-Hill, New York 1979.

[2] Hoffmann, H., Chem.-Ing.-Tech. 54 (1982) pp. 865-876.

[3] Gibilaro, L.G., Chem. Eng Sci. 33 (1978) pp. 487-492.

[4] Nauman, E.B., Chem. Eng Sci. 36 (1981) pp. $957-966$.

[5] Wen, C.Y., Fan, L.T., Models for flow systems and chemical reactors, Chemical Processing and Engineering, Vol. 3, Marcel Dekker, New York 1975.

[6] Villermaux, J., van Swaaij, W.P.M., Chem. Eng Sci. 24 (1969) pp. 1097-1111.

[7] Grabmüller, H., Schädlich, H.K., Chem. Eng Sci. 38 (1983) pp. 1543-1553.

[8] Østergaard, K., Michelsen, M.L., Can. J. Chem. Eng 47 (1969) pp. 107-112.

[9] Midoux, N., Charpentier, J.C., Chem. Eng J. (Lausanne) 1 (1970) pp. $163-167$.

[10] Michelsen, M.L., Østergaard, K., Chem. Eng Sci. 25 (1970) pp. 583-592.

[11] Anderssen, A.S., White, E.T., Chem. Eng Sci. 25 (1970) pp. $1015-1021$.

[12] Anderssen, A.S., White, E.T., Chem. Eng Sci. 26 (1971) pp. 1203-1221.

[13] Pham, Q.T., Keey, R.B., Chem. Eng Sci. 32 (1977) pp. 786-788.

[14] Hopkins, M.J., Sheppard, A.J., Eisenklam, P., Chem. Eng Sci. 24 (1969) pp. $1131-1137$.

[15] Abbi, Y.P., Gunn, D.J., Trans. Inst. Chem. Eng 54 (1976) pp. 225-231.

[16] Westerterp, K.R., van Swaaij, W.P.M., Beenackers, A.A.C.M., Chemical Reactor Design and Operation-2nd ed. John Wiley \& Sons, Chichester (UK) 1984.

[17] Kan Kin-Mun, Greenfield, P.F., AIChE J. 29 (1983) pp. 123- 132.

[18] Hochmann, J.M., Effron, E., Ind. Eng Chem. Fundam. 8 (1969) pp. 63-71.

[19] Saez, A.E., Carbonelli, R.G., AIChE J. 31 (1985) pp. 52-62.

[20] Sater, V.E., Levenspiel, O., Ind. Eng Chem. Fundam. 5 (1966) pp. 86-92.

[21] Stiegel, G.J., Shah, Y.T., Ind. Eng Chem. Process Des. Dev. 16 (1977) pp. $37-43$.

[22] Turpin, J.L., Huntington, R.L., AlChE J. 13 (1967) pp. 1196 - 1202.

[23] Weber, H.H., Dissertation, TU Darmstadt 1961.

[24] Ford, L.H., Thesis, Univ. London 1960.

[25] Achwal, S.K., Stepanek, J.B., Chem. Eng J. (Lausanne) 12 (1976) pp. $69-75$.

[26] Saada, M.Y., Period. Polytech., Chem. Eng 19 (4) (1975) pp 317-337.

[27] Otake, T., Kunigita, E., Kagaku Kogaku 22 (1958) p. 144.

[28] Levenspiel, O., Smith, W.K., Chem. Eng Sci. 6 (1957) pp. 227-233. 